C. S. Rajan · T. N. Venkataramana

\title{
On the first cohomology of arithmetic groups
}

\author{
Received: 14 September 2000 / Accepted: 6 June 2001
}

\begin{abstract}
We study the restriction to smaller subgroups, of cohomology classes on arithmetic groups (possibly after moving the class by Hecke correspondences), especially in the context of first cohomology of arithmetic groups. We obtain vanishing results for the first cohomology of cocompact arithmetic lattices in $S U(n, 1)$ which arise from hermitian forms over division algebras $D$ of degree $p^{2}, p$ an odd prime, equipped with an involution of the second kind. We show that it is not possible for a 'naive' restriction of cohomology to be injective in general. We also establish that the restriction map is injective at the level of first cohomology for non co-compact lattices, extending a result of Raghunathan and Venkataramana for co-compact lattices.
\end{abstract}

\section{Introduction}

In this paper we study the restriction to smaller subgroups, of cohomology groups of arithmetic groups, especially in the context of first cohomology of arithmetic groups. Here, "restriction" actually means restriction of the cohomology class in question, after possibly moving the class by "Hecke correspondeces" (we call this the weak restriction, see Definition 2 in Sect. (2.1)).

Our first purpose here is to obtain vanishing results for the first cohomology of cocompact arithmetic lattices in $S U(n, 1)$ which arise from hermitian forms over division algebras $D$ of degree $p^{2}, p$ an odd prime, equipped with an involution of the second kind. Our proof is a variant of a method of Oda [O], who proved that the Albanese variety of an algebraic variety of the form $\Gamma \backslash S U(n, 1) / K$ is a factor of the direct sum of the abelian varieties associated to Shimura curves. Here $\Gamma$ is an arithmetic lattice in $S U(n, 1)$ of the type shown by Kazhdan [K] to have nonvanishing first Betti number, and $K$ is a maximal compact subgroup of $S U(n, 1)$.

We first establish that the weak restriction map (see Definition 2 of Sect. (2.1)) from a compact arithmetic quotient of the unit ball in $\mathbb{C}^{n}$ to any subvariety $Z$ is injective (at the level of first cohomology). Precisely, this means the following. Let $\Gamma$ be any co-compact (torsion-free) arithmetic subgroup of $S U(n, 1)$. The symmetric

C. S. Rajan, T. N. Venkataramana: School of Mathematics, Tata Institute of Fundamental Research, Homi Bhabha Road, Bombay - 400 005, India. e-mail: rajan@math.tifr.res.in; venky@math.tifr.res.in

Mathematics Subject Classification (2000): Primary 11F75; Secondary 22E40, 22E41 
space of $S U(n, 1)$ is the unit ball $X$ in $\mathbb{C}^{n}$ with an appripriate $S U(n, 1)$ invariant metric, and the quotient $\Gamma \backslash X$ is a smooth projective variety. Suppose $Z \subset \Gamma \backslash X$ is a subvariety. Then, we prove that given a cohomology class $\alpha \in H^{1}(\Gamma, \mathbb{C})$, the "restriction" in the above sense (of a translate under a Hecke correspondence of ) the cohomology class $\alpha$ to the sub-variety $Z$ is non-zero.

We apply the above result to the following situation. Suppose $G$ is a semi-simple algebraic group defined over $\mathbb{Q}$ such that the group $G(\mathbb{R})$ of real points, is, up to compact factors, isomorphic to $S U(n, 1)$. Suppose that $H$ is a semi-simple algebraic $\mathbb{Q}$ group with a morphism $H \rightarrow G$ of $\mathbb{Q}$-groups with finite kernel. Suppose that $H(\mathbb{R})$ is isomorphic to $S U(p-1,1)$ up to compact factors, and that the inclusion of the symmetric space of $H$ into that of $G$ is holomorphic. We assume that the subgroup $H$ is of the form $R_{K / \mathbb{Q}} S U_{1}(D), R_{F / \mathbb{Q}}$ is the Weil restriction of scalars, where $D$ is a division algebra of degree $p, p$ a prime, over an imaginary quadratic extension $E$ of a totally real number field $F$ with an involution $*$ of the second kind (see Sect. (2.3)) such that the unitary group $S U(D)(F \otimes \mathbb{R})$ is a product of $U(p-1,1)$ with a compact group. By using the result stated in the last paragraph, we reduce questions on the vanishing of the first cohomology of congruence subgroups of $G(\mathbb{R})$ to that of $H(\mathbb{R})$.

A vanishing theorem for (the first cohomology of ) such lattices in the latter case was proved over $\mathbb{Q}$ by Rapoport [Rp, p.291], Rogawski [Ro] for $S U(2,1)$ and in full generality by Clozel [Cl], using the results and methods of Kottwitz in his work on the zeta functions associated to Shimura varieties. We deduce from this result, and the injectivity result, that the first cohomology group $H^{1}(\Gamma, \mathbb{C})$ is zero, for all congruence subgroups $\Gamma$ of $G(\mathbb{Q})$.

We remark here that the result on injectivity follows from a special case of a general criterion obtained in [CV, Prop. 2.2]. Our formulation however is geometric and we give a direct proof of this result.

The injectivity of the restriction map as above, is equivalent to restricting the cohomology to the family of subgroups obtained by conjugating by all the rational elements $G(\mathbb{Q})$ of the bigger group $G$. We call this the weak restriction. It is natural to ask if the restriction map is injective in a naive sense. By this we mean the natural map on the restriction of the cohomology of an arithmetic lattice $\Gamma$ contained in the ambient group $G$, to the discrete subgroup obtained by intersecting $\Gamma$ with the real points of a subgroup $H$. We show in Sect. 3, that the injectivity of the naive restriction is not possible in general even after conjugating by an arbitrary but fixed finite set of elements, once the level becomes sufficiently large. We do this by comparing the growth of the cohomology groups of $\Gamma$ and $\Gamma \cap H(\mathbb{R})$ as one goes down the level. For this we prove some estimates giving a lower bound for the multiplicities of automorphic representations when one goes down the level of congruence subgroups, and this result seems to us of independent interest.

When the lattices are no longer cocompact, it is not clear that Oda's method can be applied, since it depends on the use of Hodge theory. In this situation when the lattices are no longer cocompact, we prove an injectivity result at the level of first cohomology by relating it to the relative congruence subgroup property. Bass, Milnor and Serre [BMS, p. 135] had shown that if an arithmetic lattice $\Gamma$ satisfies the congruence subgroup property, then all the first cohomology groups of $\Gamma$ with 
algebraic coefficients vanish. Raghunathan and Venkataramana [RV] used a relative congruence subgroup property to establish that the restriction map is injective at the level of first cohomology, provided the lattices are co-compact. We extend this result to non co-compact lattices. However the presence of unipotent elements in the non co-compact case, makes the proof in the non co-compact case simpler than the proof in the co-compact case. The main ingredient in the proof is a theorem of Raghunathan giving a criteria for a generalised congruence subgroup kernel to be finite. As a corollary we obtain a theorem of Millson [Mi], that for certain congruence arithmetic lattices in $S O(n, 1)$, the first Betti number does not vanish for $n \geq 5$, by reducing it to the case of arithmetic lattices in $S U(n, 1)$, where it is known by a theorem of Wallach [Wa].

\section{The restriction map}

In this section we define the restriction map and prove Theorem 1. As a corollary we will deduce that for certain arithmetic lattices $\Gamma$ contained in $S U(n, 1), H^{1}(\Gamma, \mathbf{C})=$ $(0)$.

\subsection{Notation}

Let $G / \mathbb{Q}$ semisimple algebraic group defined over $\mathbb{Q}$, such that all the $\mathbb{Q}$-simple factors are non-compact at infinity. $G(\mathbb{Q})$ is a subgroup of $G\left(\mathbb{A}_{f}\right)$, where $\mathbb{A}_{f}$ is the ring of finite adéles of $\mathbb{Q}$. There is a natural topology on $G\left(\mathbb{A}_{f}\right)$ which makes $G\left(\mathbb{A}_{f}\right)$ into a locally compact totally disconnected topological group. Let $K \subset G\left(\mathbb{A}_{f}\right)$ be a compact open subgroup. Then $\Gamma:=K \cap G(\mathbb{Q})$ is referred to as a congruence arithmetic subgroup of $G(\mathbb{Q})$. A subgroup $\Gamma$ of $G(\mathbb{Q})$ is said to be arithmetic, if $\Gamma$ is commensurable with a congruence arithmetic subgroup of $G(\mathbb{Q})$. If $\Gamma$ is an arithmetic subgroup, then $G(\mathbb{Q})$ is the commensurator group, consisting of those elements $g \in G(\mathbb{R})$ such that $g \Gamma g^{-1}$ is commensurable with $\Gamma$.

Let $X$ denote the symmetric space associated to $G(\mathbb{R})$, the space of maximal compact subgroups of $G(\mathbb{R})$. To a point $x \in X$, we let $K_{x}$ denote the corresponding maximal compact subgroup of $G(\mathbb{R})$ with Lie algebra $\mathfrak{k}_{x}$. We have an orthogonal decomposition $\mathfrak{g}=\mathfrak{k}_{x} \oplus \mathfrak{p}_{x}$, with respect to the Killing form. We assume that $\Gamma$ is a torsion-free arithmetic lattice in $G(\mathbb{R})$. Denote by $S(\Gamma)=\Gamma \backslash X$, the corresponding manifold.

Suppose $Z$ is a manifold and we have a smooth morphism $\phi: Z \rightarrow \Gamma \backslash X$. Let $\tilde{Z} \rightarrow Z$ denote the universal cover of $Z$, and $\tilde{\phi}: \tilde{Z} \rightarrow X$ be the lift of $\phi$. We will denote by $\phi_{*}$ the corresponding morphism at the level of fundamental groups or at the level of tangent spaces, and $\phi^{*}$ the pullback map at the level of cohomology or of differential forms. For any $g \in G(\mathbb{Q})$, we denote by $Z_{g}$ the following manifold:

$$
Z_{g}:=\phi_{*}^{-1}\left(\Gamma \cap g^{-1} \Gamma g\right) \backslash \tilde{Z} .
$$

Since $\Gamma$ is an arithmetic lattice, the commensurator subgroup of $\Gamma$ is $G(\mathbb{Q})$, and hence $Z_{g}$ is a compact manifold and is a finite cover of $Z$. 
Definition 1. The restriction map is the natural map on the cohomology groups,

$$
\phi^{*}:=\phi_{\Gamma}^{*}: H^{*}(\Gamma \backslash X, F) \rightarrow H^{*}(Z, F) .
$$

Here $F$ is any field, which we usually take to be either $\mathbb{Q}$ or $\mathbb{R}$. Note that since $\Gamma \backslash X$ is a $K(\Gamma, 1)$ space, there is a natural isomorphism $H^{*}(\Gamma \backslash X, F) \simeq H^{*}(\Gamma, F)$ of the singular cohomology groups of $\Gamma \backslash X$ with the group cohomology of $\Gamma$. Suppose now that $\Gamma \subset G(\mathbb{Q})$ a congruence arithmetic subgroup. We have the natural morphisms at the level of cohomology groups,

$$
H^{*}(\Gamma \backslash X, F) \stackrel{g^{*}}{\rightarrow} H^{*}\left(g^{-1} \Gamma g \backslash X, F\right) \stackrel{\phi^{*}}{\longrightarrow} H^{*}\left(Z_{g}, F\right) .
$$

Let $\phi_{g}^{*}=\phi^{*} g^{*}: H^{*}(\Gamma \backslash X, F) \rightarrow H^{*}\left(Z_{g}, F\right)$ be the composite morphism.

Suppose now that $F=\mathbb{R}$ or $\mathbb{C}$ and that we are considering deRham cohomology groups. Let $\omega$ be a differential form representing a cohomology class in $\Gamma \backslash X$. Consider $\phi_{g}^{*}(\omega)$ as a form on the universal cover $\tilde{Z}$. Then at the level of forms we have,

$$
\phi_{g}^{*}(\omega)=\tilde{\phi}^{*}\left(g^{*} \omega\right) \text {. }
$$

Definition 2. The weak restriction map or simply Res is defined to be

$$
\operatorname{Res}:=\prod_{g \in G(\mathbb{Q})} \phi_{g}{ }^{*}: H^{*}(\Gamma \backslash X, F) \rightarrow \prod_{g \in G(\mathbb{Q})} H^{*}\left(Z_{g}, F\right) .
$$

Suppose that $X$ is a Hermitian symmetric domain. Assume now that $G$ is anisotropic over $\mathbb{Q}$. $\Gamma$ is then a cocompact arithmetic lattice and $\Gamma \backslash X$ is a compact Kähler manifold. Let $Z$ be a compact Kähler manifold and $\phi: Z \rightarrow \Gamma \backslash X$, be a holomorphic map. For a point $m \in M$ with $M$ a complex manifold, denote by $T_{m} M$ the holomorphic tangent space to $M$ at $m$. With this notation we now have the following version of Oda's principle.

Theorem 1. Let $X$ be a Hermitian symmetric domain and $\Gamma$ be a cocompact arithmetic lattice in $G(\mathbb{Q})$. Let $Z$ be a compact Kähler manifold and $\phi: Z \rightarrow \Gamma \backslash X$, be a holomorphic map. Fix an integer $q \geq 0$. Assume now that $\phi$ satisfies the following: there exists a point $y_{0} \in Z$ such that the $K_{\tilde{\phi}\left(y_{0}\right)}$ span of $\tilde{\phi}_{*}\left(\wedge^{q}\left(T Z_{y_{0}}\right)\right)$ is equal to $\wedge^{q}\left(T X_{\tilde{\phi}\left(y_{0}\right)}\right)$.

Then we have an injection of cohomology groups

$$
\text { Res : } H^{q, 0}(\Gamma \backslash X) \rightarrow \prod_{g \in G(\mathbb{Q})} H^{q, 0}\left(Z_{g}\right) .
$$

Proof. It is a consequence of the Kähler identities for the $d$ and the $\bar{\partial}$ Laplacians on a compact Kahler manifold, that the holomorphic forms inject into the deRham cohomology groups of a compact Kähler manifold. Hence if a holomorphic form vanishes as a cohomology class, then the form is identically zero. Suppose now $\omega$ is a holomorphic $q$-form representing a cohomology class in $H^{q}(\Gamma \backslash X, \mathbf{C})$, belonging to the kernel of Res. Then for any $g \in G(\mathbb{Q}), \phi_{g}^{*}(\omega)$ considered as a holomorphic 
form on $Z_{g}$ is identically zero. At the level of the morphism $\tilde{Z} \rightarrow X$, this translates to

$$
\tilde{\phi}^{*}\left(g^{*} \omega\right)=0, \quad \text { for all } g \in G(\mathbb{Q}) .
$$

Now $G(\mathbb{Q})$ is dense in $G(\mathbb{R})$, and the action of $G(\mathbb{R})$ on the space of forms on $X$ by conjgation is a continuous action. Hence it follows

$$
\tilde{\phi}^{*}\left(g^{*} \omega\right)=0, \quad \text { for all } g \in G(\mathbb{R}) .
$$

In particular, we have that if $\xi \in \tilde{\phi}_{*}\left(\wedge^{q} T Z_{\tilde{\phi}\left(y_{0}\right)}\right)$, then $g^{*}(\omega)(\xi)=0$. By our hypothesis that the $K_{y_{0}}$ span of $\tilde{\phi}_{*}\left(\wedge^{q} T Z_{y_{0}}\right)$ is the whole of the holomorphic tangent space $\wedge^{q}\left(T X_{\tilde{\phi}\left(y_{0}\right)}\right)$, we obtain that

$$
g^{*}(\omega)(\xi)=0, \forall g \in G(\mathbb{R}), \forall \xi \in \wedge^{q}\left(T X_{\tilde{\phi}\left(y_{0}\right)}\right) .
$$

Now let $x$ be any point on $X$ and $\xi$ be a $q$-multivector at $x$. We can find an element $g \in G(\mathbb{R})$, such that $g x=y_{0}$. Then $\omega(\xi)=g^{*}(\omega)\left(g_{*}(\xi)\right)=0$. Hence we have shown that $\omega=0$.

Remark. We remark that we have made no mention of special points in the statement of the theorem, in contrast to the approach by [O] and [MR] (of course, [MR] obtain other information, like fields of definition etc, by considering special points). However, it is clear that the arithmeticity of $\Gamma$ is an essential ingredient in the proof, in that we require the commensurability group of $\Gamma$ to be dense in $G(\mathbb{R})$. We will see in the next section that it is not possible to obtain injectivity for the restriction map in the strong sense, even after taking finitely many conjugates.

Corollary 1. With assumptions as in the theorem above, we have that the restriction map

$$
\text { Res }: H^{1}(\Gamma \backslash X, \mathbb{C}) \rightarrow \prod_{g \in G(\mathbb{Q})} H^{1}\left(Z_{g}, \mathbb{C}\right)
$$

is an injection.

Proof. This follows from the Hodge decomposition for a compact Kähler manifold $M$,

$$
H^{1}(M, \mathbb{C})=H^{0,1}(M) \oplus H^{1,0}(M), \overline{H^{0,1}}(M)=H^{1,0}(M),
$$

and the fact that a holomorphic map preserves the type of a form.

Corollary 2. Suppose that the symmetric space associated to $G(\mathbb{R})$ is isomorphic to the symmetric space associated to $S U(n, 1)$. Let $\Gamma$ be a cocompact arithmetic lattice and let $\phi: Z \rightarrow \Gamma \backslash X$ be a holomorphic map from a compact Kahler manifold $Z$ of rank $r$ at some point $y_{0} \in Z$. Then for any $q \leq r$,

$$
\text { Res : } H^{q, 0}(\Gamma \backslash X) \rightarrow \prod_{g \in G(\mathbb{Q})} H^{q, 0}\left(Z_{g}\right)
$$

is an injection. 
Proof. This follows from the fact that for the group $S U(n, 1)$, a maximal compact subgroup is $S(U(n) \times U(1))$, and the associated action on the $q^{\text {th }}$ exterior power of the holomorphic tangent space is the action of $S U(n)$ on $\wedge^{q} \mathbb{C}^{n}$. This latter action is irreducible for $q \leq n$.

\subsection{Injectivity of restriction for compact quotients of the unit ball}

We are now interested in applying Theorem 1 to the following situation. Let $G$ be a connected, semi-simple, simply connected group defined and anisotropic over $\mathbb{Q}$. Write $G=\prod_{i=1}^{r} G_{i}$, where each $G_{i}$ is a $\mathbb{Q}$-simple, connnected, simply connected group. Assume as before that $G_{i}(\mathbb{R})$ is not compact for each $i$. A congruence arithmetic subgroup $\Gamma$ of $G(\mathbb{Q})$ is commensurable with a product $\prod_{i=1}^{r} \Gamma_{i}$, where $\Gamma_{i} \subset G_{i}(\mathbb{Q})$ is a congruence arithmetic subgroup. Thus the first Betti number of $\Gamma$ can be computed in terms of the Betti numbers of $\Gamma_{i}(1 \leq i \leq r)$. Hence we may assume that $G$ is $\mathbb{Q}$-simple, and that $G(\mathbb{R})$ is non-compact. If $G(\mathbb{R}) \neq \operatorname{Spin}(n, 1)$ or $S U(n, 1)$ upto compact factors, then it is well known from the work of Matsushima, Kaneyuki, Nagano, Kazhdan, Kostant, Bernstein [BoW, p. 168] that

$$
H^{1}(\Gamma, \mathbb{C})=0
$$

for all congruence arithmetic subgroups. Therefore we will henceforth assume that upto compact factors $G(\mathbb{R})=\operatorname{Spin}(n, 1)$ or $S U(n, 1)$.

Suppose $H$ is a connected, semi-simple, simply connected group over $\mathbb{Q}$, which is $\mathbb{Q}$-simple. Suppose $j: H \rightarrow G$ is a $\mathbb{Q}$-rational morphism of algebraic groups with finite kernel. It is then easy to see that $H$ is anisotropic over $\mathbb{Q}$ as well. Assume also that $H(\mathbb{R})$ is noncompact. Let $X$ and $Y$ denote respectively the symmetric spaces associated to $G(\mathbb{R})$ and $H(\mathbb{R})$. We denote by $\left.\Gamma \cap g^{-1} \Gamma g \cap H(\mathbb{R})\right)$ the subgroup $j_{\mathbb{R}}^{-1}\left(\Gamma \backslash g^{-1} \Gamma g\right)$ of $H(\mathbb{R})$. In this situation we have the following special case of Corollary 2. Note that in the situation below, $\left(\Gamma \cap g^{-1} \Gamma g \cap H(\mathbb{R})\right) \backslash Y$ is a manifold.

Theorem 2. Suppose $G(\mathbb{R})$ (resp. $H(\mathbb{R}))$ are $S U(n, 1)$ (resp. $S U(p, 1))$ upto compact factors (then $X_{H}$ and $X_{G}$ are Hermitian symmetric domains). Assume that the natural map $j_{\mathbb{R}}: X_{H} \rightarrow X_{G}$ is holomorphic. Let $\Gamma \subset G(\mathbb{R})$ be a cocompact, torsion-free lattice in $G(\mathbb{R})$. Then

$$
\text { Res }: H^{1}(\Gamma \backslash X, \mathbb{C}) \rightarrow \prod_{g \in G(\mathbb{Q})} H^{1}\left(\left(\Gamma \cap g^{-1} \Gamma g \cap H(\mathbb{R})\right) \backslash Y, \mathbb{C}\right)
$$

is injective.

\subsection{A vanishing result}

Our purpose now is to obtain a vanishing result for the first cohomology of certain types of arithmetic lattices in $S U(n, 1)$, by the method of restriction of cohomology. We use and extend the vanishing results for the first cohomology of Rapoport, Rogawski and Clozel. Suppose $F$ is a totally real number field of degree $r$ over 
$\mathbb{Q}$, and let $E$ be a totally imaginary quadratic extension of $F$. Let $\sigma$ denote the non-trivial element of the Galois group of $E$ over $F$. Let $D$ be a central division algebra of degree $p^{2}, p$ an odd prime, over $E$ of the second kind with involution $\iota$, such that $\iota(z)=\sigma(z)$ for all $z \in E \subset D$. Consider the special unitary group defined over $F$,

$$
H_{F}=S U(D)=\left\{x \in D^{*}, N_{\text {red }}(x)=1 \mid x \iota(x)=1\right\},
$$

where $N_{\text {red }}(x)$ denotes the reduced norm of $x \in D^{*}$. Let

$$
H:=\underset{F / \mathbb{Q}}{\operatorname{Res}}\left(H_{F}\right)
$$

be the Weil restriction of scalars. Let $\Delta \subset H(\mathbb{Q})$ be a congruence arithmetic subgroup. We have the following theorem, which was proved in some special cases initially by Rapoport [Rp], by Rogawski [Ro] when $p=3$ and by $\mathrm{Clozel}[\mathrm{Cl}]$ when $p$ is an odd prime.

Theorem 3 (Rapoport, Rogawski, Clozel). With the foregoing notation,

$$
H^{1}(\Delta, \mathbb{C})=0 .
$$

Consider now a left vector space $V$ of dimension $k$ over $D$, together with a hermitian form $B$ with respect to the involution $i$. Let $G(B)$ be the Weil restriction of scalars of the special isometry group of this hermitian form. We assume that the form is such that $G(B)(\mathbb{R})=S U(k p-1,1) \times S U(k p)^{r-1}$.

Example. Choose a basis $\left\{e_{1}, e_{2}, \cdots, e_{k}\right\}$ of $V$ over $D$. Fix a set $\tau_{1}, \tau_{2}, \cdots, \tau_{r}$ of distinct non-conjugate embeddings of $E$ into $\mathbb{C}$. We continue to denote by $\tau_{i}$ the map which sends an element $x \in D^{*}$ to $x \otimes 1 \in D \otimes_{E, \tau_{i}} \mathbb{C}$. With respect to any of the embeddings, we have that $D \otimes_{E, \tau_{i}} \mathbb{C} \simeq M(p, \mathbb{C})$, and the involution $\iota$ can be conjugated to the standard involution $x \rightarrow \bar{x}^{t}$. The space of matrices $A \in M(p, \mathbb{C})$ satisfying $\iota(A)=A$, such that the unitary group of the hermitian form $B_{A}(x)=\iota(x) A x$ is of type $U(p-i, i), 0 \leq i \leq p$ is open. Since the space $\{a \in D \mid \iota(a)=a\}$ is a subspace of $D$ defined over $F$, by weak approximation we can find an element $a \in D^{*}$ (resp. $b \in D^{*}$ ), such that the isometry group of the hermitian form $B_{i}(x)=\iota(x) \tau_{i}(a) x, x \in M(p, \mathbb{C})$ is of type $U(p-1,1)$ (resp. of type $U(p, 0))$ for $i=1$ and of type $U(p, 0)$ otherwise.

Let $B_{a}(x)=\iota(x) a x, x \in D\left(\operatorname{resp} . B_{b}(x)=\iota(x) b x, x \in D\right)$ denote the corresponding hermitian forms on $D$. Then $G\left(B_{a}\right)(\mathbb{R}) \simeq S U(p-1,1) \times S U(p)^{r-1}$ and $G\left(B_{b}\right)(\mathbb{R}) \simeq S U(p) \times S U(p)^{r-1}$. Write now an element $x \in V$ as $x=\sum_{l=1}^{k} x_{l} e_{l}$. Let $B(x)$ denote the hermitian form $B(x)=B_{a}\left(x_{1}\right)+\sum_{l=2}^{k} B_{b}\left(x_{l}\right)$. Then we have that $G(B)(\mathbb{R}) \simeq S U(k p-1,1) \times S U(k p)^{r-1}$.

Let $\Gamma$ be a congruence arithmetic subgroup contained in $\operatorname{Res}_{F / \mathbb{Q}}(G)(\mathbb{R})$. As a corollary of Theorem 2 and Theorem 3, we obtain

Theorem 4. With notation as above,

$$
H^{1}(\Gamma \backslash X, \mathbb{C})=0 .
$$




\section{Non-injectivity of naive restriction}

In this section we show that it is not possible to obtain an injectivity of the restriction map on the cohomology groups, when one works with a naive notion of restriction. More precisely our aim is to show the following:

Theorem 5. Let $S$ be a finite subset of $G(\mathbb{Q})$, and $G, H$ be anisotropic $\mathbb{Q}$ groups with a finite morphism $H \rightarrow G$ of $\mathbb{Q}$-algebraic groups as in section 1 , with the symmetric spaces $X_{G}$ and $X_{H}$ associated to $G$ and $H$ being of Hermitian type, and such that the map $X_{H} \rightarrow X_{G}$ is holomorphic. Assume morever that $G(\mathbb{R})$ (resp. $H(\mathbb{R}))$ is locally isomorphic to $S U(n, 1) \times S U(n+1)^{r-1}($ resp. $S U(1,1) \times$ $\left.S U(2)^{r-1}\right)$. Suppose $n>3 r$. Then the following restriction map

$$
R_{\Gamma, S}: H^{1}(\Gamma \backslash X, \mathbb{C}) \rightarrow \prod_{g \in S} H^{1}\left(\left(\Gamma \cap g^{-1} \Gamma g \cap H(\mathbb{R})\right) \backslash Y, \mathbb{C}\right)
$$

cannot be injective for all congruence subgroups $\Gamma \subset G(\mathbb{Q})$.

Proof. The method of proof is to compare the growth of the cohomology groups as one goes down the level of $\Gamma$. For a congruence arithmetic lattice $\Gamma$ corresponding to a compact open subgroup $K \subset G\left(\mathbb{A}_{f}\right)$, let $m\left(\pi_{\infty} \otimes \pi_{f}\right)$ be the multiplicity with which the representation $\pi_{\infty} \otimes \pi_{f}$ occurs in $L^{2}(\Gamma(\mathbb{Q}) \backslash G(\mathbb{A}))$. We recall the Matsushima formula $[\mathrm{BoW}]$ :

$$
H^{1}(\Gamma \backslash X, \mathbb{C})=\bigoplus m\left(\pi_{\infty} \otimes \pi_{f}\right) H^{1}\left(\mathfrak{g}, \mathfrak{k}, \pi_{\infty}\right) \otimes \pi_{f}^{K}
$$

Note that $G$ is assumed to be anisotropic over $\mathbb{Q}$, hence the Matsushima formula is valid as stated. Let $\pi=\pi_{\infty} \otimes_{p} \pi_{p}$ be a cuspidal automorphic representation of $G(\mathbb{A})$, such that the archimedean component contributes to the relative Lie algebra cohomology $H^{1}\left(\mathfrak{g}, \mathfrak{k}, \pi_{\infty}\right) \neq 0$. We note that for us, the symbol $\otimes_{p}$ is a restricted tensor product. Let $p$ be such that $G\left(\mathbb{Q}_{p}\right)$ is non-compact. Then we note that if $\pi_{p}$ is the " $p$-adic" component of $\pi$ then $\pi_{p}$ cannot be finite dimensional: for, otherwise, this would mean that $\pi_{p}$ is one dimensional, and then by a well known argument using strong approximation, that $\pi_{\infty}$ is also one dimensional, but the trivial representation of $G(\mathbb{R})$ does not have non-trivial first cohomology as can be easily seen. Choose a compact open subgroup $K \subset G\left(\mathbb{A}_{f}\right)$ such that $\pi_{f}^{K} \neq 0$. We may further assume that $K=\prod K_{p}$ where the product is over all primes $p$ and $K_{p} \subset G\left(\mathbb{Q}_{p}\right)$ is a compact open subgroup. For a prime $p$, write $K^{p}=\prod K_{l}$ where $l$ runs through all primes except $p$.

Fix now a prime $p$ such that $G / \mathbb{Q}_{p}$ contains a factor locally isomorphic to $S L_{n+1}$ over $\mathbb{Q}_{p}$. We will choose a sequence of compact subgroups $K_{m} \subset K \subset G\left(\mathbb{A}_{f}\right)$ for $m$ large enough, with $K_{m}=K^{p} K_{p}(m)$, where $K^{p}=K \cap \prod_{l \neq p} G\left(\mathbb{Q}_{l}\right)$, and $K_{p}(m)$ denotes the principal congruence subgroups of level $m$ at the prime $p$, i.e., the kernel of the reduction modulo $p^{m}$ of the group $G L_{n}\left(\mathbb{Z}_{p}\right)$,

$$
K_{p}(m)=\operatorname{Ker}\left(G L_{n}\left(\mathbb{Z}_{p}\right) \rightarrow G L_{n}\left(\mathbb{Z} / p^{m} \mathbb{Z}\right)\right) .
$$


Let $\Gamma_{m}:=G(\mathbb{Q}) \cap K_{m}$ be the corresponding congruence subgroup inside $G(\mathbb{Q})$. It follows from the Matsushima formula that the first Betti number $h^{1}\left(\Gamma_{m}\right)$ of $\Gamma_{m}$ satisfies the following estimate:

$$
h^{1}\left(\Gamma_{m}\right) \geq \operatorname{dim}\left(\pi_{f}^{K_{m}}\right) \geq \operatorname{dim}\left(\pi_{p}^{K_{p}(m)}\right) .
$$

We now recall a fact about the growth of invariants (under smaller and smaller open compact subgroups of $G_{p}$ ) of an admissible representation $\pi_{p}$ of a linear $p$-adic group $G_{p}$ over $\mathbb{Q}_{p}$. Let

$$
D=\min \frac{1}{2} \operatorname{dim} \mathcal{O},
$$

where $\mathcal{O}$ runs over the collection of positive dimensional nilpotent orbits of $G_{p}$ in the adjoint representation on the Lie algebra of $G_{p}$. The following proposition is a consequence of the theorems of Howe for $G L_{n}$ and Harish-Chandra for general $G$, on the germ expansion of characters of admissible representations. We refer to [DP, Prop. 1, p. 189], and for a proof of the proposition, to [Sa2, p. 143].

Proposition 1. Let $\pi$ be an irreducible infinite dimensional admissible representation of $G_{p}$. Let $K_{p}(m)$ be the principal congruence subgroup of level $m$. Then there exists a positive constant $c>0$, such that for $m$ large enough,

$$
\operatorname{dim}\left(\pi_{p}^{K_{p}(m)}\right) \geq c p^{m D} .
$$

It is easy to check that for $G$ locally isomorphic to $S L_{n+1}$ we have $D=n$. Hence it follows from combining the Proposition with the above estimate for the Betti number coming from the Matsushima formula, that for $m$ large enough,

$$
h^{1}\left(\Gamma_{m} \backslash X\right) \geq c_{1} p^{m n},
$$

for some positive constant $c_{1}$.

Note that the same proof shows the following result, which seems to us to be of independent interest.

Theorem 6. Let $G$ be a semi-simple algebraic group over $\mathbb{Q}$ such that $G(\mathbb{R})$ is noncompact. Let $\pi$ be an irreducible infinite dimensional representation of $G(\mathbb{R})$ which occurs discretely in $L^{2}(\Gamma \backslash G(\mathbb{R}))$ for some congruence subgroup $\Gamma$. Fix a prime $p$ such that $G\left(\mathbb{Q}_{p}\right)$ is noncompact. Let $\Gamma\left(p^{m}\right)$ be the principal congruence subgroup of $\Gamma$ of level $p^{m}$. Then the multiplicity of $\pi$ in the discrete part of $L^{2}\left(\Gamma\left(p^{m}\right) \backslash G(\mathbb{R})\right)$ is bounded below by a constant times $p^{m D}$ where $D$ is half the dimension of the minimal positive dimensional nilpotent orbit in the Lie algebra of $G$.

Restricting our attention now to $H$, we observe first that there is an asymptotic upper bound [Sa1], given by $d\left(\pi_{\infty}^{H}\right) \operatorname{vol}\left(g^{-1} \Gamma g \cap H(\mathbb{R}) \backslash H(\mathbb{R})\right)$, for the multiplicity with which a representation occurs in $L^{2}\left(g^{-1} \Gamma g \backslash H(\mathbb{R})\right)$. Here we are fixing a Haar measure on the group $H(\mathbb{R})$ and $d\left(\pi_{\infty}^{H}\right)$ is the formal degree of $H(\mathbb{R})$-representation $\pi_{\infty}^{H}$ with respect to this measure. Since the number of representations of $H(\mathbb{R})$ which have non-trivial cohomology (with trivial coefficients) 
is finite, it follows that the growth of the cohomology group $H^{1}\left(g^{-1} \Gamma g \cap H(\mathbb{R})\right)$ is bounded above by,

$$
h^{1}\left(g^{-1} \Gamma g \cap H(\mathbb{R})\right) \leq c_{2} \operatorname{vol}\left(g^{-1} \Gamma g \backslash H(\mathbb{R})\right),
$$

where $c_{2}$ is some absolute constant. Since for every $g \in S$, we have assumed that $g \in \tilde{G}\left(\mathbb{Z}_{p}\right)$, we have that the elements of $S$ normalise $K_{p}(m)$. Hence the growth of the volume as a function of $m$, is given by

$$
c_{3}|S|\left|\left(K_{p}(0) \cap \tilde{H}\left(\mathbb{Z}_{p}\right): K_{p}(m) \cap \tilde{H}\left(\mathbb{Z}_{p}\right)\right)\right| .
$$

From the structure of $K_{p}(0)$ it follows that

$$
\left|\left(K_{p}(0) \cap \tilde{H}\left(\mathbb{Z}_{p}\right): K_{p}(m) \cap \tilde{H}\left(\mathbb{Z}_{p}\right)\right)\right|=\left|\tilde{H}\left(\mathbb{Z} / p^{m} \mathbb{Z}\right)\right| .
$$

We have a filtration of the group,

$$
\tilde{H}\left(\mathbb{Z} / p^{m} \mathbb{Z}\right) \rightarrow \tilde{H}\left(\mathbb{Z} / p^{m-1} \mathbb{Z}\right) \rightarrow \cdots \tilde{H}(\mathbb{Z} / p \mathbb{Z}) \rightarrow\{1\},
$$

where the kernels except the last one, can be identified with the vector space $\tilde{\mathfrak{h}}\left(\mathbb{F}_{p}\right)$, where $\tilde{\mathfrak{h}}$ denotes the Lie algebra of $\tilde{H}$. Suppose that $H(\mathbb{R})$ is locally isomorphic to $S U(1,1) \times S U(2)^{r-1}$. Then $\operatorname{dim}(\tilde{\mathfrak{h}})=3 r$, and $\left|\tilde{H}\left(\mathbb{Z} / p^{m} \mathbb{Z}\right)\right|=\left|\tilde{H}\left(\mathbb{F}_{p}\right)\right| p^{3 r(m-1)}$. From all this it follows that

$$
\operatorname{dim}\left(\prod_{g \in S} H^{1}\left(\left(\Gamma \cap g^{-1} \Gamma_{m} g \cap H(\mathbb{R})\right) \backslash Y, \mathbb{C}\right)\right) \leq c_{4} p^{3 r m}
$$

for some constant $c_{4}$. But then this does not grow as fast as the cohomology group of $H^{1}\left(\Gamma_{m} \backslash X, \mathbb{C}\right)$, provided we assume that $n>3 r$.

Remark. It is clear from the proof that we could have assumed $H(\mathbb{R})$ to be locally isomorphic to $S U(k, 1) \times S U(k+1)^{r-1}$, provided the inequality $r\left((k+1)^{2}-1\right)<n$ holds.

\section{Congruence subgroup property and first Betti number}

Our aim in this section is to prove that the weak restriction map is injective at the level of first cohomology when the lattices are no longer co-compact. For cocompact lattices this result was proved in [RV]. Even when both the groups involved are unitary groups, Oda's method cannot be applied directly as it rests on the use of Hodge theory.

Let $G, H$ be connected, simply connected $\mathbb{Q}$-groups, which are $\mathbb{Q}$-simple, with a morphism $j: H \rightarrow G$ of $\mathbb{Q}$ algebraic groups. The group $G(\mathbb{Q})$, can be equipped with two topological group structures, obtained by defining an arithmetic (resp. congruence) subgroup of $G(\mathbb{Q})$ to be an open subgroup. These topological structures will be respectively called the arithmetic and the congruence topologies on $G(\mathbb{Q})$. The group $G(\mathbb{Q})$ admits a completion with respect to these topologies in the 
sense of [Bour], and we will denote by $\widehat{G(\mathbb{Q})} a$ (resp. $\widehat{G(\mathbb{Q})_{c}}$ ) the arithmetic (resp. congruence) completions of $G(\mathbb{Q})$. We have the following exact sequence,

$$
1 \rightarrow C(G) \rightarrow \widehat{G(\mathbb{Q})}_{a} \stackrel{\pi}{\rightarrow} \widehat{G(\mathbb{Q})}_{c} \rightarrow 1,
$$

where $\pi$ is induced by the identity map on $G(\mathbb{Q})$. Here $C(G)$ is the congruence subgroup kernel and is a profinite group. The group $C(G)$ being trivial, amounts to saying that every arithmetic subgroup is a congruence subgroup. The congruence subgroup problem is the determination of the congruence subgroup kernel. In particular the question is to know when $C(G)$ is a finite group. It was shown in [BMS], [Ra1, page 153], that if $C(G)$ is finite, then $H^{1}(\Gamma, \rho)=0$, for any finite dimensional representation of $\Gamma$. In $[R V]$, a relative version of the congruence subgroup property was established, and used to show the injectivity of the restriction map on cohomology provided the lattices are cocompact.

\subsection{Notation}

Let $\Gamma \subset G(\mathbb{Q})$ be a congruence arithmetic subgroup. Then $g^{-1} \Gamma g \cap H(\mathbb{R})=\Delta_{g}$ is a congruence arithmetic subgroup of $H(\mathbb{Q})$. Analogous to the restriction maps defined in Section 2, we can define restriction maps at the group cohomology level as follows:

Definition 3. Define the map $j^{*}$ as the restriction map

$$
j^{*}:=j_{\Gamma}^{*}: H^{*}(\Gamma, \mathbb{Q}) \rightarrow H^{*}\left(j^{-1}(\Gamma), \mathbb{Q}\right) .
$$

If $g \in G(\mathbb{Q})$ and $\Gamma \subset G(\mathbb{Q})$ a congruence arithmetic subgroup, then we have an isomorphism $g^{*}: H^{*}(\Gamma, \mathbb{Q}) \rightarrow H^{*}\left(g^{-1} \Gamma g, \mathbb{Q}\right)$, induced by $\gamma \rightarrow g^{-1} \gamma g$ on $\Gamma$. Let

$$
j_{g}^{*}: H^{*}(\Gamma, \mathbb{Q}) \stackrel{g^{*}}{\rightarrow} H^{*}\left(g^{-1} \Gamma g, \mathbb{Q}\right) \stackrel{j^{*}}{\rightarrow} H^{*}\left(g^{-1} \Gamma g \cap H, \mathbb{Q}\right)
$$

be the composite morphism $j^{*} g^{*}$.

Definition 4. The restriction map or simply Res is defined to be

$$
\operatorname{Res}:=\prod_{g \in G(\mathbb{Q})} j_{g}^{*}: H^{*}(\Gamma, \mathbb{Q}) \rightarrow \prod_{g \in G(\mathbb{Q})} H^{*}\left(g^{-1} \Gamma g \cap H(\mathbb{R}), \mathbb{Q}\right) \text {. }
$$

\subsection{Relative congruence subgroup kernel}

Fix a congruence subgroup $\Gamma \subset G(\mathbb{Q})$, and $g \in G(\mathbb{Q})$. Then

(i) the closure of $\Gamma$ in $\widehat{G(\mathbb{Q})_{a}}$ is precisely the profinite completion $\hat{\Gamma}$ of $\Gamma$, and $C(G) \subset \hat{\Gamma}$.

(ii) let $\hat{\Gamma}_{c}$ be the closure of $\Gamma$ in $\left.\widehat{G(\mathbb{Q})}\right)_{c}$. Then one has the exact sequeunce

$$
1 \rightarrow C(G) \rightarrow \hat{\Gamma}_{a} \rightarrow \hat{\Gamma}_{c} \rightarrow 1
$$


One can similarly define $C(H),{\widehat{H(\mathbb{Q})_{a}}}_{a}$, and $\widehat{H(\mathbb{Q})}_{c}$. The map $j_{g}: g^{-1} \Gamma g \cap$ $H(\mathbb{R}) \rightarrow g^{-1} \Gamma g$ induces the following commutative diagram:

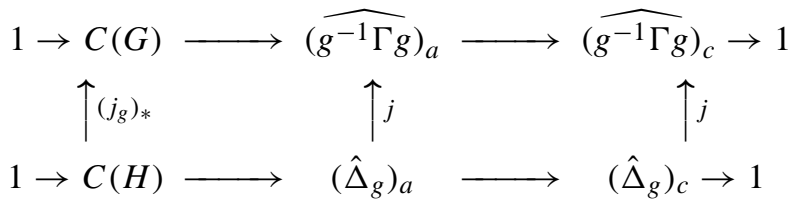

It may be checked that for $g \in G(\mathbb{Q})$, Image $\left(j_{g}\right)_{*}=g^{-1} \operatorname{Image}\left(j_{1}\right)_{*} g$. Let $N_{H}$ be the closed subgroup of $C(G)$ generated by

$$
\left\langle g^{-1} j_{1 *}\left(C_{H}\right) g \mid g \in G(\mathbb{Q})\right\rangle .
$$

Define the relative congruence subgroup kernel

$$
C(G, H)=C(G) / N_{H} .
$$

As has been observed in [RV], $C(G, H)$ is related to the restriction map Res : $H^{1}(\Gamma, \mathbb{C}) \rightarrow \prod H^{1}\left(g^{-1} \Gamma g \cap H(\mathbb{R}), \mathbb{C}\right)$.

Proposition 2. If $C(G, H)$ is finite or even torsion, then

$$
\text { Res : } H^{1}(\Gamma, \mathbb{C}) \rightarrow \prod H^{1}\left(g^{-1} \Gamma g \cap H(\mathbb{R}), \mathbb{C}\right)
$$

is injective.

\subsection{A Theorem of Raghunathan}

The main ingredient in the proof of our theorem is a theorem of Raghunathan giving a criterion for a generalised congruence subgroup kernel to be finite. In this section we assume from now onwards that $G, H$ are isotropic over $\mathbb{Q}$ and that

$$
\operatorname{rank}_{\mathbb{R}}(G)=\operatorname{rank}_{\mathbb{R}}(H)=\operatorname{rank}_{\mathbb{Q}}(G)=\operatorname{rank}_{\mathbb{Q}}(H)=1 .
$$

Let $S \simeq G_{m}$ be a maximal $\mathbb{Q}$-split torus in $H$. Then $A=j(S)$ is also a maximal $\mathbb{Q}$-split torus in $G$.

The $\mathbb{Q}$ group $G$ is in particular an algebraic variety, and we denote by $\operatorname{dim}(G)$ its Zariski dimension. Denote by $\pm \alpha, \pm 2 \alpha$ the non-zero roots of $A$ acting on the Lie algebra of $G$. Denote by $U^{+}=U_{\alpha}$ the subgroup of $G$ corresponding to the Lie subalgebra of the Lie algebra of $G$, which is the direct sum of the $\alpha$ root space and the $2 \alpha$-root space. Let $U^{-}=U_{-\alpha}$ be the subgroup of $G$ corresponding to the Lie subalgebra which is the direct sum of the $-\alpha$ and $-2 \alpha$ root spaces. Put $U_{H}^{ \pm}=U^{ \pm} \cap H$. Then, $U^{ \pm}$are maximal unipotent subgroups of $G$.

Let $M$ denote the centralizer of $A$ in $G$. It is known that the action of $M$ on the $\alpha$-root space is irreducible, being isomorphic upto twisting by a character to the standard representation of the orthogonal or unitary groups.

Let $N \subset C(G)$ be a closed subgroup of the congruence subgroup kernel $C(G)$ of $G$, normalised by $G(\mathbb{Q})$. Hence it will also be normalised by $\widehat{G(\mathbb{Q})_{a}}$. Let $C=$ 
$C(G) / N$. The closure of $U^{ \pm}(\mathbb{Q}) \subset G(\mathbb{Q})$ in the group $\widehat{G(\mathbb{Q})_{a}}$ is isomorphic to $U^{ \pm}\left(\mathbb{A}_{f}\right)$, since $\pi$ splits over $U^{ \pm}\left(\mathbb{A}_{f}\right)$ [Ra1], [Ra2]. We recall that $\pi$ is the natural map from $\widehat{G(\mathbb{Q})_{a}}$ onto $\widehat{G(\mathbb{Q})_{c}}=G\left(\mathbb{A}_{f}\right)$. Let

$$
\widehat{G(\mathbb{Q})_{N}}=\widehat{G(\mathbb{Q})_{a}} / N \text {. }
$$

Via $\pi^{-1}$ we get sections $\sigma^{ \pm}: U^{ \pm}\left(\mathbb{A}_{f}\right) \rightarrow \widehat{G(\mathbb{Q})_{a}} \rightarrow \widehat{G(\mathbb{Q})_{N}}$. Let $\hat{G}_{p}$ be the group generated by $\sigma^{+}\left(U^{+}\left(\mathbb{Q}_{p}\right)\right)$ and $\sigma^{-}\left(U^{-}\left(\mathbb{Q}_{p}\right)\right)$ in $\widehat{G(\mathbb{Q})}{ }_{N}$. We have the following theorem of Raghunathan [Ra1], [Ra2]:

Theorem 7 (Raghunathan [Ra1], [Ra2], [Ra3]).

(i) If $C$ is central in $\widehat{G(\mathbb{Q})}{ }_{N}$, then $C$ is finite.

(ii) Iffor every pair $(p, q)$ of distinct primes the groups $\hat{G}_{p}$ and $\hat{G}_{q}$ commute, then $C$ is central in $\widehat{G(\mathbb{Q})_{N}}$.

Proof. i) follows from the fact that the Pontrjagin dual of $C$ injects into the metaplectic kernel, and the metaplectic kernel is known to be finite [Ra3]. ii) is a restatement of [Ra2, Prop. 2.14].

\subsection{Injectivity of restriction in the non-co-compact case}

We have the following theorem extending the results of [RV] to the case when $\Gamma$ is no longer a co-compact lattice.

Theorem 8. With assumptions as above, assume also that the image Lie $(j(H)) \cap$ $u_{\alpha} \neq 0$ where $u_{\alpha}$ is the $\alpha$ root space. Then $C(G, H)$ is finite and

$$
\text { Res : } H^{1}(\Gamma, \mathbb{C}) \rightarrow \prod H^{1}\left(g^{-1} \Gamma g \cap H(\mathbb{R}), \mathbb{C}\right)
$$

is injective.

Proof. In view of Proposition 2, we need only show that $C(G, H)$ is finite. For $N_{H}$ as in Subsection 4.2, let

$$
\widehat{G(\mathbb{Q})_{\mathrm{rel}}}=\widehat{G(\mathbb{Q})_{a}} / N_{H}
$$

By Theorem 7, it is sufficient to show that

$$
1 \rightarrow C(G, H) \rightarrow \widehat{G(\mathbb{Q})_{\mathrm{rel}}} \stackrel{\pi}{\rightarrow} \widehat{G(\mathbb{Q})_{c}} \simeq G\left(\mathbb{A}_{f}\right) \rightarrow 1
$$

is a central extension. This will be deduced by verifying the condition (ii) of Theorem 7. Since the image of the congruence subgroup kernel of $H$ is trivial in $\widehat{G(\mathbb{Q})_{\text {rel }}}$, it follows that for any pair of distinct primes $p$ and $q$,

$$
\left[U_{H}^{+}\left(\mathbb{Q}_{p}\right), U_{H}^{-}\left(\mathbb{Q}_{q}\right)\right]=1 .
$$

The natural embedding of $G(\mathbb{Q})$ inside $\widehat{G(\mathbb{Q})_{a}}$ gives a splitting $\tau: G(\mathbb{Q}) \rightarrow$ $\widehat{G(\mathbb{Q}}_{\text {rel }}$, compatible with the embedding of $G(\mathbb{Q})$ inside $G\left(\mathbb{A}_{f}\right)$. Suppose $m \in$ 
$M(\mathbb{Q})$, and $u_{p} \in U\left(\mathbb{Q}_{p}\right)$. The density of $U(\mathbb{Q})$ in $U\left(\mathbb{Q}_{p}\right)$ and the compatibility of

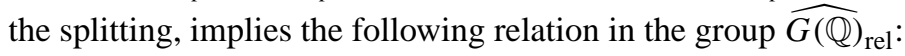

$$
\operatorname{Ad}(\tau(m))\left(\sigma\left(u_{p}\right)\right)=\sigma\left(\operatorname{Ad}\left(m_{p}\right)\left(u_{p}\right)\right)
$$

where $m_{p}$ denotes the element $m$ thought of as an element in $M\left(\mathbb{Q}_{p}\right)$. Hence we have for $u_{p} \in U_{H}^{+}\left(\mathbb{Q}_{p}\right), u_{q} \in U_{H}^{-}\left(\mathbb{Q}_{q}\right)$

$$
\operatorname{Ad}(\tau(m))\left(\left[\sigma\left(u_{p}\right), \sigma\left(u_{q}\right)\right]\right)=\left[\sigma\left(\operatorname{Ad}\left(m_{p}\right)\left(u_{p}\right)\right), \sigma\left(\operatorname{Ad}\left(m_{q}\right)\left(u_{q}\right)\right)\right]=1 .
$$

By weak approximation the diagonal subgroup $M(\mathbb{Q})$ is dense in the product $M\left(\mathbb{Q}_{p}\right) \times M\left(\mathbb{Q}_{q}\right)$.

Since we have assumed that $\operatorname{Lie}(j(H))$ intersects the $\alpha$ root space of $G$ nontrivially, we see from the irreducibility of the $M$ action on the $\alpha$-root space, that the elements $A d\left(m_{p}\right)\left(u_{p}\right)$ generate the group $\sigma\left(U^{+}\left(\mathbb{Q}_{p}\right)\right)$ inside $\widehat{G(\mathbb{Q})_{\text {rel }}}:$ let $V\left(\mathbb{Q}_{p}\right)$ denote the subgroup of $U\left(\mathbb{Q}_{p}\right)$ so generated. First, the projection of $V\left(\mathbb{Q}_{p}\right)$ to the abelianisation of $U_{\alpha}\left(\mathbb{Q}_{p}\right)$ (which is isomorphic to the vector space of $\mathbb{Q}_{p}$ rational points of the $\alpha$ root space) is surjective, by irreducibility. Secondly, any subgroup of $U_{\alpha}\left(\mathbb{Q}_{p}\right)$ which maps onto the abelianisation of $U_{\alpha}\left(\mathbb{Q}_{p}\right)$ is all of $U_{\alpha}\left(\mathbb{Q}_{p}\right)$, as can be easily seen. Thus, $V\left(\mathbb{Q}_{p}\right)$ is the same as $U_{\alpha}\left(\mathbb{Q}_{p}\right)$.

We note that an analogous argument is already used in [Ra2] (cf. the proof of Lemma (3.2) of [Ra2]), in the course of the proof of the Congruence Subgroup Property.

Hence we obtain,

$$
\left[\sigma\left(U^{+}\left(\mathbb{Q}_{p}\right)\right), \sigma\left(U^{-}\left(\mathbb{Q}_{q}\right)\right)\right]=1 .
$$

By virtue of the splitting $\sigma$ of $U^{ \pm}\left(\mathbb{A}_{f}\right)$, we obtain that the commutator

$$
\left[\sigma\left(U^{+}\left(\mathbb{Q}_{p}\right)\right), \sigma\left(U^{+}\left(\mathbb{Q}_{q}\right)\right)\right]=1 .
$$

Since $U^{ \pm}\left(\mathbb{Q}_{p}\right)$ generate $\hat{G}_{p}$, we have

$$
\left[\hat{G}_{p}, \hat{G}_{q}\right]=1,
$$

and that proves the theorem.

Remark. It can be seen from the proof of the theorem that in the non co-compact case, the presence of the unipotent groups $U^{ \pm}$makes it easier to check Raghunathan's criterion for the relative congruence subgroup kernel to be finite. Thus the proof in the non-cocompact case turns out to be easier than the corresponding result proved in $[\mathrm{RV}]$ for the co-compact case.

It was shown by Wallach [Wa], that if $\Gamma$ is a non co-compact congruence subgroup in $S U(n, 1)$, then there is a subgroup $\Gamma^{\prime}$ of finite index in $\Gamma$, such that $H^{1}(\Gamma, \mathbb{Q}) \neq 0$. As a corollary, it follows from the structure theory of non cocompact arithmetic lattices in $S O(n, 1), n \geq 3$, that we have an embedding $j: S O(n, 1) \rightarrow S U(n, 1)$ over $\mathbb{Q}$, with $\operatorname{Lie}(j(H))$ intersecting the $\alpha$ root space $u_{\alpha}$ non-trivially. We thus have the following special case of a theorem of Millson [Mi]. 
Corollary 3. If $\Gamma \subset S O(n, 1)$ is a non co-compact congruence arithmetic subgroup, then $H^{1}\left(\Gamma^{\prime}, \mathbb{Q}\right) \neq 0$, for some congruence arithmetic subgroup $\Gamma^{\prime}$ of $\Gamma$.

Acknowledgements. The possibility of proving the vanishing of first Betti number in Theorem 4 was suggested to us by M. S. Raghunathan; we also thank him for very useful conversations on the use of the congruence subgroup kernel in the proof of Corollary 3.

We are grateful to Jean-Pierre Labesse for pointing out a mistake in our proof of Theorem 5 . He had already considered the idea of estimating growth of Betti numbers using the growth of invariants of open subgroups of $S L_{n+1}\left(\mathbb{Q}_{p}\right)$. We are also grateful to Dipendra Prasad for providing a reference to Proposition 1 . We thank the referee for a very careful reading of the manuscript and for some very relevant corrections and remarks.

\section{References}

[BMS] Bass, H., Milnor, J. and Serre, J.-P.: Solution of the congruence subgroup problem for $S L_{n}(n \geq 3)$ and $S p_{2 n}(n \geq 2)$. Publ. Math. I.H.E.S. 33, 59-137 (1967)

[Bour] Bourbaki, N.: Éléments de Mathématique, Topologie Générale. Chapitre 3, Paris: Hermann, 1951

[BoW] Borel, A. and Wallach, N.R.: Continuous cohomology, discrete subgroups and representations of reductive groups. Annals of Math. Studies 94, Princeton, NJ: Princeton Univ. Press, 1980

[Ca] Cartier, P.: Representation theory of p-adic groups, A survey. Automorphic forms, representations and $L$-functions, Proc. Symp. Pure Math. vol. 33, Part 1, Providence, R.I.: Amer. Math. Soc., 1977, pp. 111-155

[Cl] Clozel, L.: On the cohomology of Kottwitz's arithmetic varieties. Duke Math. J. 72, 757-795 (1993)

[CV] Clozel, L. and Venkataramana, T.N.: Restriction of the holomorphic cohomology of a Shimura variety to a smaller Shimura subvariety. Duke Math. J. 95, 51-106 (1998)

[Cor] Automorphic forms, representations and $L$-functions. (A. Borel and W. Casselman, eds.) Proc. Symp. Pure Math. vol. 33, Parts 1 and 2, Providence, R.I.: Amer. Math. Soc., 1977

[K] Kazhdan, D.: Some applications of the Weil representation. J. d'Analyse Math. 32, 235-248 (1977)

[Mi] Millson, J.J.: On the first Betti number of a constant negatively curved manifold. Ann. Math. 104, 235-247 (1970)

[MR] Murty, V.K. and Ramakrishnan, D.: The Albanese of unitary Shimura varieties. In: The Zeta functions of Picard modular surfaces, eds. R.P. Langlands and D. Ramakrishnan, Les Publications CRM, Montreal, 1992, 445-460

[O] Oda, T.: A note on the Albanese variety of an arithmetic quotient of the complex hyperball. J. Fac. Sci. Univ. Tokyo, Sect. 1A Math. 28, 481-486 (1981)

[DP] Prasad, D.: A brief survey on the theta correspondence. In: Number Theory, eds. V. Kumar Murty and M. Waldschmidt, Contemp. Math. 210, Providence RI: Amer. Math. Soc., 1998, pp. 171-193

[Ra1] Raghunathan, M.S.: On the congruence subgroup problem. Publ. Math. Inst. Hautes Etud. Sci. 46, 107-161 (1976)

[Ra2] Raghunathan, M.S.: On the congruence subgroup problem II. Invent. Math. 85, 73-117 (1986) 
[Ra3] Raghunathan, M.S.: The congruence subgroup problem. In: Proceedings of the Hyderabad conference on algebraic groups, ed. S. Ramanan, National Board for Higher Mathematics, Mumbai, 1991, pp. 465-494

[RV] Raghunathan, M.S. and Venkataramana, T.N.: The first Betti number of arithmetic groups and the congruence subgroup problem. In: Linear algebraic groups and their representations (Los Angeles, CA, 1992), Contemp. Math. 153, Providence, RI: Amer. Math. Soc., 1993, pp. 95-107

[Rp] Rapoport, M.: On the bad reduction of Shimura varieties. In: Automorphic forms, Shimura varieties, and $L$-functions: Proceedings of a Conference held at Ann Arbor, Michigan, Vol. II, eds. L. Clozel and J. S. Milne, Perspectives in Mathematics, 11, New York: Academic Press, 1990

[Ro] Rogawski, J.D.: Automorphic representations of unitary groups in three variables. Annals of Math. Studies, 123, Princeton, NJ: Princeton University Press

[Sa1] Savin, G.: Limit multiplicities of cusp forms. Invent. Math. 95, no. 1, 149-159 (1989)

[Sa2] Savin, G.: Dual pair $G_{\mathcal{J}} \times P G L_{2} G_{\mathcal{J}}$ is the automorphism group of the Jordan algebra $\mathcal{J}$. Inv. Math. 118, 141-160 (1994)

[Wa] Wallach, N.R.: Square integrable automorphic forms and cohomology of arithmetic quotients of $\mathrm{SU}(p, q)$. Math. Ann. 266, no. 3, 261-278 (1984) 